\title{
Safety and image quality of cardiovascular magnetic resonance imaging in patients with retained epicardial pacing wires after heart transplantation
}

\author{
Constantin Gatterer ${ }^{1,2}\left(\mathbb{0}\right.$, Marie-Elisabeth Stelzmüller ${ }^{3}$, Andreas Kammerlander ${ }^{2}$, Andreas Zuckermann ${ }^{3}$ (D),
}

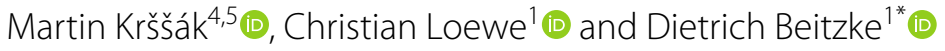

\begin{abstract}
Background: Temporary epicardial pacing wires, implemented in patients during heart transplantation, are routinely removed before discharge. However, in some cases, these wires may remain in situ and are often considered as a contraindication for cardiovascular magnetic resonance (CMR) imaging in the future. Therefore, we aimed to provide data about safety and image quality of CMR in these patients.

Methods: This is a report on a subpopulation out of 88 patients after heart transplantation that were included in a prospective cohort study and underwent multiple CMR in their post-transplant course. During CMR, patients were monitored by electrocardiogram and all examinations were observed by a physician to document potential adverse events. Additionally, image quality was assessed by an imaging specialist.

Results: Nineteen of 88 patients included had temporary pacing wires in situ. These patients underwent a total of 51 CMR studies. No major adverse event and only one single, mild sensory event could be documented. All CMR studies showed preserved diagnostic image quality. Temporary pacing wires were visible in 100\% of HASTE and cine sequences. In less than 50\% of the examinations, temporary pacing wires were also visible in T1 and T2 mapping, short tau inversion recovery (STIR), and late gadolinium enhancement (LGE) sequences, without any impairment of image quality.

Conclusions: With a low event rate of only one mild adverse event during 51 CMR examinations (2\%), CMR appears to be safe in patients with retained temporary epicardial pacing wires after heart transplantation. Moreover, image quality was not impaired by the presence of pacing wires.
\end{abstract}

Keywords: Cardiovascular magnetic resonance, MR safety, Cardiac transplantation, Image quality

\section{Introduction}

Patients after heart transplantation are at high risk for post-operative bradyarrhythmia, including

\footnotetext{
${ }^{*}$ Correspondence: dietrich.beitzke@meduniwien.ac.at

${ }^{1}$ Department of Biomedical Imaging and Image-guided Therapy, Division

of Cardiovascular and Interventional Radiology, Medical University

of Vienna, Währinger Gürtel 18-20, 1090 Vienna, Austria

Full list of author information is available at the end of the article
}

atrioventricular block and sinus node dysfunction [1]. Thus, temporary pacing wires are routinely inserted in the right atrium (RA) and right ventricle (RV) after successful implantation of the donor's heart. Furthermore, intermittent temporal pacing can facilitate the patients' weaning process from cardiopulmonary bypass [2]. During the postoperative course, these wires are intended to be completely removed. However, in some 
cases, the wires have to be fixed at the myocardium (e.g. fixation by a suture to stop bleeding at the time of the insertion) or cannot be removed due to pericardial adhesion or overlap with sutures of the fascia. In these cases, pacing wires are usually cut below the skin level.

With these wires attached to the heart, patients are often excluded from magnetic resonance imaging (MRI) in the future [3]. As MRI utilization continuously increases and has a substantial impact on patient care, excluding these patients from MRI might potentially hinder a diagnostic work-up in the future $[4,5]$.

Causes for concern relate to potential neuromuscular voltage induction that could result in arrhythmias and movement, or heating of pacing wires with consequent tissue damage [6-8]. Preclinical data indicates that the greatest risk arises from the radiofrequency (RF) field due to the circularly polarized magnetic field associated with MRI devices. Higher amounts of energy may be deposited in the patient's body by RF via abandoned pacemaker (PM) leads or pacing wires, resulting in heat burns and potentially inducing arrhythmic events $[8$, 9]. However, even using higher systemic absorption rates ex vivo, no myocardial damage could be observed so far [10]. Also there are several studies on patients undergoing MRI with retained temporary pacing wires published the that do not report any adverse events [3, 11-14]. However, the MR systems and scan regions in these reports were quite heterogeneous, and thus, did not allow a systematic comparison on this topic. To date, data about the safety of cardiovascular magnetic resonance (CMR) have been described only in a case report [12].

MRI and CMR with perrmanent pacemaker (PPM) systems including subcutaneous PPM generators is already a well-established procedure and as nowadays most of these systems are approved for CMR/MRI under specified conditions by the vendors [15]. In contrary to permanent systems temporary wires did not undergo systematic testing for being MR conditional as these "temporary" pacing wires are not intended to remain in the patient. Additionally, temporary pacing wires are not implanted in a standardized way, and therefore the shape and the length of the abandoned wires differ from patient to patient making it impossible to predict potential CMR interaction in the further course.

Another important aspect of CMR in contrast to other imaging regions is image quality when dealing with temporary pacing wires and conventional PPM remnants, as leads can significantly alter image quality due to artifacts [16]. Especially in CMR, these artifacts may conceal pathologies and could potentially influence diagnostic image quality, as well as quantitative values of mapping techniques.
Therefore, the aim of this study was to provide further evidence about the safety and image quality in a patient cohort with temporary pacing wires who were referred to multiparametric CMR in a prospective setting.

\section{Methods}

Patients:

This subgroup analysis is part of an ongoing, prospective CMR cohort study of 100 patients after heart transplantation. It complies with the declaration of Helsinki and its amendments and was approved by the ethics committee of the Medical University of Vienna (IRB Nr.: 2012/2015). The inclusion criteria were: patients 18-99 years; and recent cardiac transplantation. Exclusion criteria were defined as the presence of abandoned implanted cardiodefibrilator (ICD) leads, claustrophobia, and pregnancy. Written, informed consent was obtained from each participating patient prior to study inclusion. The study was performed at a tertiary referral center that offered the entire spectrum of cardiovascular imaging and an organ transplant program, as part of EuroTransplant, which performs approximately 50 to 60 heart transplantations annually. Within this prospective cohort study, patients are scheduled for CMR 3, 12, 24, 36, 48 and 60 months after heart transplantation.

During heart transplantation surgery, temporary pacing wires (TME bifurcated, OSYPKA AG, Rheinfelden, Germany) were implanted at the RA and RV. The leads were then loosely folded to a loop and led intrapericardially to the xiphoid where they penetrated the skin to be connected to the extracorporeal PM. Epicardial wires that could not be removed were left in situ after being cut below the skin level. Patients who participated in this prospective cohort study and had retained temporary pacing wires were briefed about reporting any skin perceptions or heat sensations during the CMR examination.

Using routinely performed chest X-ray, computed tomography, and fluoroscopy, retained epicardial pacing wires were categorized according to their shape into "straight", "C-shaped," or "looped (antenna)."

All CMR examinations were performed on a $1.5 \mathrm{~T}$ CMR scanner (Avanto Fit, Siemens Healthineers, Erlangen, Germany) with a maximum gradient strength of 45 $\mathrm{mT} / \mathrm{m}$ and a maximum slew rate of $200 \mathrm{~T} / \mathrm{m} / \mathrm{s}$. Specific absorption rate (SAR) limits for normal operation mode were $2 \mathrm{w} / \mathrm{kg}$ and $4 \mathrm{w} / \mathrm{kg}$ for first level mode, respectively. Two receive only coils (18-channel body array/32-channel integrated coil) were used. A standardized multiparametric CMR protocol was used to assess myocardial inflammation. The protocol included left ventricular structure and function, advanced tissue characterization by $\mathrm{T} 1$ and $\mathrm{T} 2$ mapping, conventional edema imaging, and 
Table 1 Cardiovascular Magnetic Resonance (CMR) sequence pre-settings at 1.5T

\begin{tabular}{|c|c|c|c|c|c|c|c|c|}
\hline Sequence & TI (ms) & TE/TR (ms) & Triggerpulse & $\begin{array}{l}\text { Echo } \\
\text { spacing } \\
\text { (ms) }\end{array}$ & Flip angle $\left(^{\circ}\right)$ & Resolution (mm) & $\begin{array}{l}\text { Layer- } \\
\text { thickness } \\
(\mathrm{mm})\end{array}$ & $\begin{array}{l}\text { Readout } \\
\text { bandwith (Hz/ } \\
\text { Pixel) }\end{array}$ \\
\hline $\begin{array}{l}\text { bSSFP Cine } \\
\text { (SAx, 4Ch, 2Ch) }\end{array}$ & & $1.21 / 52.2$ & & 2.9 & 64 & $1.4 \times 1.4$ & 6 & 930 \\
\hline $\mathrm{T}_{2} \mathrm{HASTE}$ & & $43 / 467$ & $\times 2$ & 3.32 & 160 & $1.6 \times 1.6$ & 5 & 781 \\
\hline $\begin{array}{l}T_{1} \text {-mapping }(S A x, 4 c h) \\
R R>700 \mathrm{~ms})\end{array}$ & 180 & $1.13 / 279.84$ & & 2.7 & 35 & $1.4 \times 1.4$ & 8 & 1085 \\
\hline $\begin{array}{l}\mathrm{T}_{1}-\text { mapping }(\mathrm{SAx} / 4 \mathrm{Ch}) \\
{ }^{*} \mathrm{RR}<700 \mathrm{~ms}\end{array}$ & 180 & $1.01 / 263.55$ & & 2.4 & 35 & $1.9 \times 1.9$ & 8 & 1085 \\
\hline $\mathrm{T}_{2}$-mapping & & 27/193.27 & & 2.5 & 70 & $1.9 \times 1.9$ & 8 & 1184 \\
\hline STIR & 150 & $47 / 760$ & $\times 2$ & 6.74 & 180 & $1.5 \times 1.5$ & 8 & 235 \\
\hline Rest Perfusion (SAx) & 115 & 1.07/193.72 & & 2.2 & & & & \\
\hline LGE (SAx,4Ch,3Ch, 2Ch) & $\begin{array}{l}(250) \\
\text { variable }\end{array}$ & $1.19 / 700$ & & 2.9 & 50 & $1.5 \times 1.5$ & 8 & 781 \\
\hline $\begin{array}{l}\text { Post-contrast } \mathrm{T}_{1} \text {-mapping } \\
\quad(\mathrm{SAx}, 4 \mathrm{Ch}) \\
{ }^{*} \mathrm{R} R>700\end{array}$ & 260 & $1.13 / 359.84$ & & 2.68 & 35 & $1.4 \times 1.4$ & 8 & 1085 \\
\hline $\begin{array}{l}\text { Post-contrast } \mathrm{T}_{1} \text {-mapping } \\
(\mathrm{SAx}, 4 \mathrm{Ch}) \\
\mathrm{RR}<700\end{array}$ & 260 & $1.01 / 241.12$ & & 2.43 & 35 & $1.9 \times 1.9$ & 8 & 1085 \\
\hline
\end{tabular}

*Heart rate adapted; bSSFP, balanced steady state free precession; SAx, short axis; $4 C h$, four chamber; $3 C h$, three chamber; $2 C h$, two chamber; $R R$, RR-Interval (ECG); $S T I R$, short tau inversion recovery; $L G E$, late gadolinium enhancement; $T I$, inversion time; $T E$, repetition time; $T R$, repetition time

scar assessment by late gadolinium enhancement (LGE) [17]. Details on the CMR protocol are listed in Table 1.

All examinations were supervised by a physician and possible adverse reactions were documented. Patients were monitored using the CMR integrated electrocardiogram (ECG) gating device. Severe adverse events were defined as arrhythmias, self-reported heat sensations, hemodynamic instability, or any other possibly life-threatening complication. Mild adverse events were defined as every other event that did not meet the criteriafor a severe adverse event and was clinically associated with a potential lead/CMR interaction. Furthermore, each CMR examination was assessed for image quality by two CMR specialists.

\section{Statistics:}

Descriptive statistics were performed for patient characteristics, image quality, and events. Continuous variables were reported as mean \pm standarddeviation if normally distributed, or as median \pm interquartile range for non-normally distributed continuous variables. Categorical variables were described as counts and percentages. Cohen's kappa was used to report interrater variability of image quality and visibility between two blinded readers.

\section{Results}

\section{Patient characteristics}

Overall, 88 patients were included in this ongoing prospective cohort study by July 2020 . Nineteen of these 88 patients (21.6\%) had retained temporary pacing wires in situ ( $42 \%$ female/ $58 \%$ male). Eleven patients had both the RA and the RV pacing wires in situ, eight patients only had the RA wire in situ. The reasons for heart transplantation in this sub-group were ischemic cardiomyopathy $(\mathrm{n}=7)$, dilative cardiomyopathy $(\mathrm{n}=9)$, Anderson-Fabry disease $(\mathrm{n}=1)$, amyloidosis $(\mathrm{n}=1)$, and valvular disease $(\mathrm{n}=1)$. Most patients had repetitive CMR examinations (5 patient had four, 6 patients had three and 5 had two examinations respectively). One patient only had a single CMR examination at the time of analysis. Overall, a total of 51 CMR examinations were analyzed for safety and image quality. The median time from heart transplantation until the first CMR was 96 days (interquartile range: 88-119 days).

In one patient, the short tau inversion recovery (STIR) sequence was not added to the protocol. In another patient, LGE and post-contrast T1 mapping sequences were interrupted due to the onset of an allergic reaction to the contrast agent. All other scans were completed according to the study protocol.

Visibility of temporary pacing wires and CMR image quality Using chest X-ray, temporary pacing wires leads were visible in only 10 of 19 patients (53\%). In eight patients, the leads were detected by chest or cardiac computed tomography $(\mathrm{CT})$ scans, while, in one patient, the existence of retained wires was only self-reported, but could not be verified on any radiological images prior to CMR. 
The most frequent lead form, derived from other imaging modalities, was a loop (47\%), followed by C-shaped (32\%), and straight (21\%). An overview of lead forms is displayed in Table 2. Figure 1 shows a representative cases of a $\mathrm{C}$-shaped and looped retained pacing wire, hardly visible on chest X-Ray.

Image quality was of diagnostic quality in all CMR studies. Although retained pacing wires were visible in all half Fourier single-shot turbo spin-echo (HASTE) sequences and $78 \%$ of cine sequences during CMR, diagnostic image quality was not degraded. Image quality in T1/T2 mapping sequences, and LGE images was also not impaired, as the wires could be detected in only $45 \%$ and $40 \%$, respectively. A detailed overview of image quality and artifacts can be found in Table 3. Examples of image quality and artifacts are shown in Fig. 2.

A good interrater agreement was found for all measures, ranging from $68 \%$ for mapping sequences to $82 \%$ for cine imaging (kappa from 0.46 to $0.57, \mathrm{p}<0.001$ for all). Both readers agreed that leads did not impair image quality in any of the cases. An overview of agreement and kappa can be found in Table 4.

\section{Safety/adverse events}

During 51 CMR examinations, no serious adverse event, such as arrhythmias, self-reported heating of the pacing wires, or severe pain, could be observed. One patient, with a C-shaped temporary pacing wire in situ, described a sensory event near the subcutaneous end of the retained lead during the second CMR,
Table 2 Overview of patient characteristics, frequency and form of retained temporary pacemakers leads, and events

\begin{tabular}{ll}
\hline Characteristic & $\mathrm{n}=19(\%)$ \\
\hline Retained temporary PM leads, $\mathrm{n}(\%)$ & $19 / 88^{*}(22)$ \\
Age, year & $54 \pm 15$ \\
Male patients, n (\%) & $11(58)$ \\
CMR Studies & $51(91)$ \\
Form & \\
Loop & $9(47)$ \\
C-shaped & $6(32)$ \\
Straight & $4(21)$ \\
Adverse reactions & \\
Severe & $0(0)$ \\
Mild & $1(5)$ \\
Event rate & \\
CMR, n (\%) & $1 / 51(2)$ \\
\hline
\end{tabular}

Numbers are given as mean \pm standard deviation or count and (\%)

*Total heart transplantation CMR study cohort; PM, pacemaker

12 months after heart transplantation. This event occurred during the HASTE sequence. The effect was reproducible, as it immediately disappeared once the sequence was discontinued, but returned as soon as it was restarted. No arrhythmic event or signs of skin irritation were observed. The CMR scan was stopped and the patient was transferred out of the CMR unit. The further course was uneventful; however, the patient was excluded from the study. All other CMR studies were completed uneventfully. With only one event of 51 CMR, the event rate was $2 \%$.

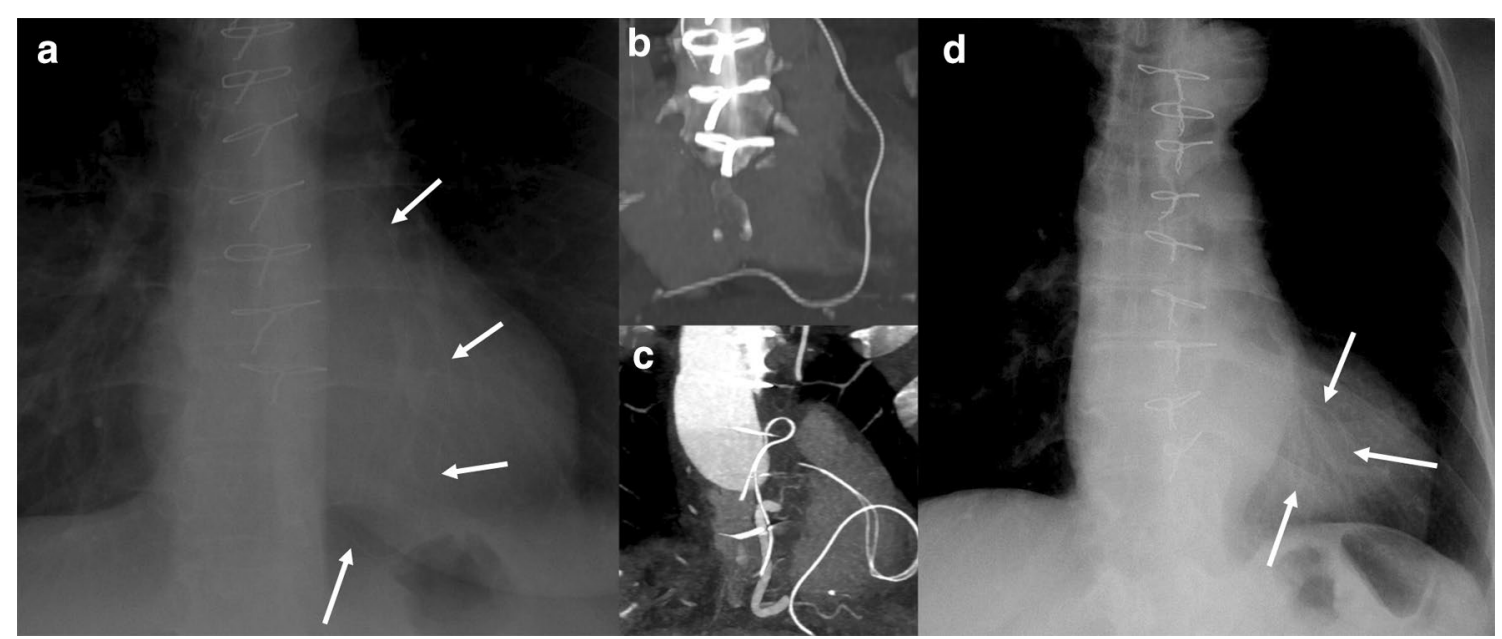

Fig. 1 Representative chest $x$-ray $(\mathbf{a}, \mathbf{d})$ and maximum intensity projection images computed tomography $(\mathrm{CT})(\mathbf{b}$, c) images of patients with retained temporary wires ( $C$ shaped configuration in $\mathbf{a}, \mathbf{b}$; looped configuration in $\mathbf{c}$, $\mathbf{d}$. Note the impaired visibility of the wires (white arrows) on the conventional chest X-Rays 


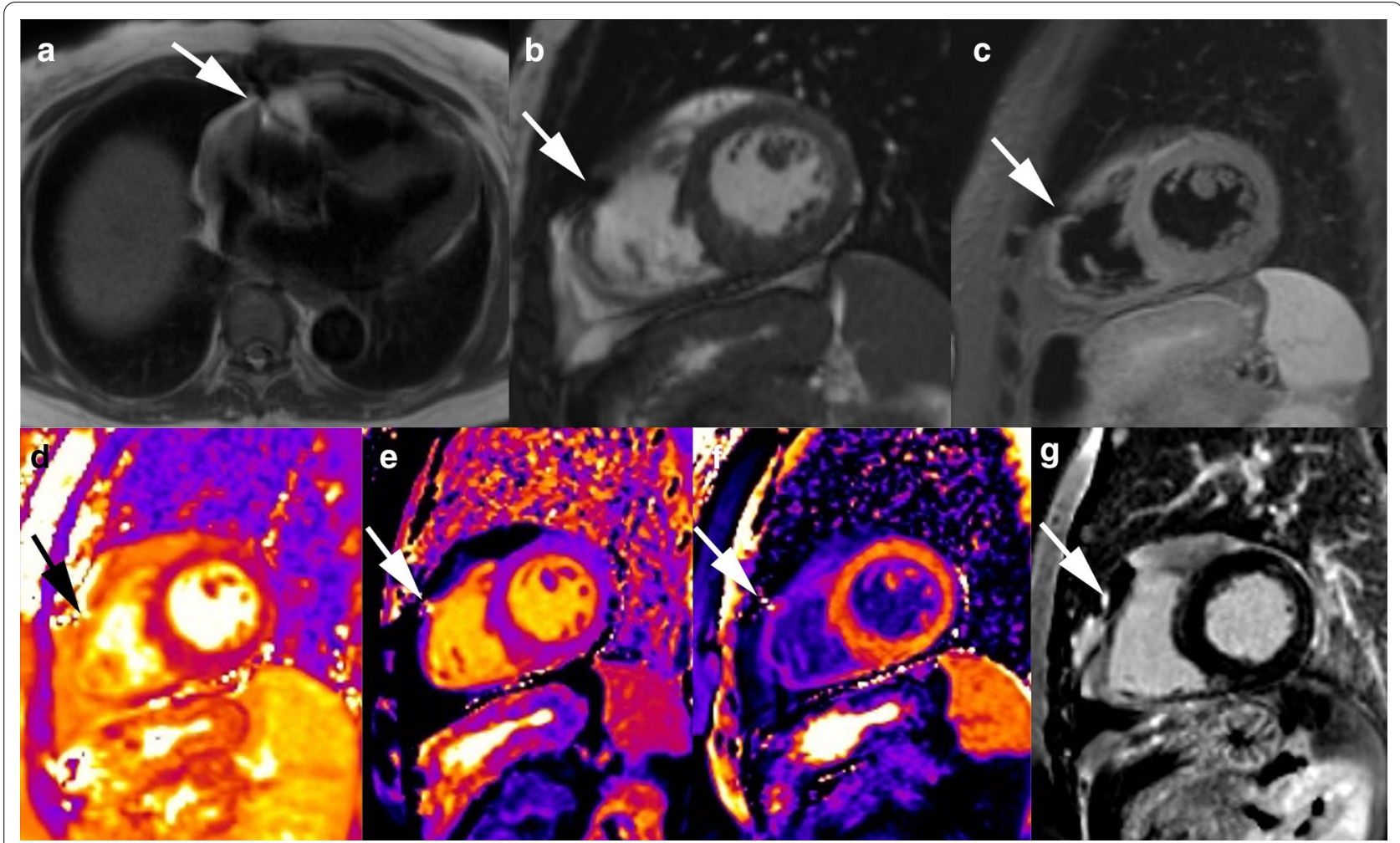

Fig. 2 Preserved image quality of CMR in a patient with retained wires (white arrows). None of the sequences are obscured by artifacts (a-g)

Table 3 Visibility and image quality of temporary Pacemaker leads in CMR

\begin{tabular}{|c|c|c|c|c|c|}
\hline Grading of image quality & $\begin{array}{c}n=51(\%) \\
\text { HASTE }\end{array}$ & $\begin{array}{l}n=51(\%) \\
\text { Cine }\end{array}$ & $\begin{array}{l}\mathrm{n}=51(\%) \\
\mathrm{T}_{1} / \mathrm{T}_{2} \text { mapping }\end{array}$ & $\begin{array}{l}n=50(\%) \\
\text { STIR }\end{array}$ & $\begin{array}{l}n=48(\%) \\
L G E\end{array}$ \\
\hline Not visible & $0(0)$ & $11(22)$ & $23(45)$ & $18(36)$ & $14(29)$ \\
\hline Visible, no image quality impairment & $51(100)$ & $40(78)$ & $23(45)$ & $23(46)$ & $19(40)$ \\
\hline Visible, impaired image quality & $0(0)$ & $0(0)$ & $0(0)$ & $0(0)$ & $0(0)$ \\
\hline Not in field of view & $0(0)$ & $0(0)$ & $5(10)$ & $9(18)$ & $15(31)$ \\
\hline
\end{tabular}

Numbers are given as count and (\%). HASTE, half-Fourier single shot turbo spin echo, LGE, late gadolinium enhancement; STIR, short tau inversion recovery

Table 4 Cohen's Kappa for interrater variability of image quality

\begin{tabular}{llll}
\hline Measure & Agreement & Kappa & $p$ \\
\hline Cine & $82 \%$ & 0.48 & $<0.001$ \\
Maps & $68 \%$ & 0.46 & $<0.001$ \\
STIR & $80 \%$ & 0.57 & $<0.001$ \\
LGE & $71 \%$ & 0.52 & $<0.001$ \\
\hline
\end{tabular}

\section{Discussion}

The main finding of this study is that multiparametric $\mathrm{CMR}$ at $1.5 \mathrm{~T}$ in patients with retained temporary pacing wires is associated with a low incidence rate of $2 \%$ mild adverse events. During a total number of $51 \mathrm{CMR}$ scans in 19 patients, not a single serious adverse event was observed. Furthermore, the image quality of CMR is not altered by retained temporary pacing wires.

MRI and, in particular, CMR utilization is continuously rising, with an increase in use and an increasing number of clinical indications for all patient groups, not only in patients after cardiac transplantation [18, 19]. The use of MRI is reported to have an important impact on clinical decision-making, with added value for patient management [4].

Patients after cardiac surgery and, especially after cardiac transplantation, often present with metallic 
remnants, either after incomplete ICD lead extraction or with temporary pacing wires as in our cohort [20]. As a consequence, patients with retained wires are often prohibited from receiving MRI, thus possibly facing worse outcomes $[3,21]$. Retained temporary pacing wires do not represent a regular medical implant, as the leads are intended to be removed after the operation. Therefore, these pacing wires are not tested for MR compatibility in a defined MR environment. In addition, the form of the wires within their epicardial course varies between patients, making it difficult to exactly predict MR-related complications. A further cause of concern may be the positioning of the patient with the wire in the center of the gradient and radiofrequency field as necessary in CMR. Thus the induction of heating and current might be more likely as if compared to other scan regions [22].

Overall, potential risks arise from interactions with multiple components of the MR system that should be taken into account separately prior to imaging in these patients. The static magnetic field (B0) may interfere with ferromagnetic implants by inducing translational force and torque. However according to the manufacturer the wires used in our cohort do not contain ferromagnetic components [23].

Relevant heating may be caused by the RF field resulting in thermal injury [23]. Kappus et al. showed that, in contrast to transvenous ICD and pacemaker leads, for which MR related heating up to $53.6^{\circ} \mathrm{C}$ was described ex vivo, no relevant heating of looped and/or straight temporary pacing wires could be found by in vitro testing using an infrared camera $[9,24]$. Furthermore, an $e x$ vivo CMR study of 12 swine hearts with implanted pacing wires provided no evidence of thermal damage to the myocardium, as evaluated in the histological examination after the CMR examination [10]. In this context it is also important that only a receive coil is used for standard CMR as the potential for heat induction over the wire is higher using a transmit/receive coil located close to the imaging center [23].

The time varying gradient magnetic field may be associated with (minor) heating of implants and neuromuscular excitation that may present as arrhythmic cardiac events [23]. Lead length and configuration, amplitude and phase of the electric field along the lead influence the current, which is potentially induced into the wires and eventually could lead to current induction, as well as additional heat elevation of the retained leads $[8,25$, 26]. This theoretical risk of arrhythmia induction might even be higher if the retained wire is still connected to the RV and/or in case of a cardiac structuralabnormality like a scar formation [23]. However, there are no reports available on arrhythmia induction by MR in the presence of retained pacing wires. Additionally, it may also be debatably whether a routine CMR protocol is able to induce voltages beyond the pacing thresholds for the atrial and ventricular leads respectively [27, 28]. These pacing thresholds are reported to increase over the time and therefore a $(\mathrm{C}) \mathrm{MR}$ far after implantation may pose a lower risk for the patient [27].

Our findings are in line with previous reports that MRI and CMR with temporary pacing wires does not lead to serious lead related complications, thus supporting the hypothesis that CMR is safe in patients after heart surgery $[3,11-14,29]$.

To date, only one case of an adverse reaction during CMR in this context has been reported in the literature. The patient, who experienced anginal symptoms during stress CMR, fully recovered and his symptoms were thought to be primarily caused by his underlying ischemic cardiomyopathy [13]. Therefore, and based on the data from this cohort, MRI, and particularly CMR, can safely be performed despite the presence of temporary right atrial and right ventricular pacing wires, with very low risk for these patients [30].

There are multiple case studies published, that describe non-MRI-related migration of the wires into various regions, including the right coronary artery, which caused an infarct, and into the ascending aorta [31, 32]. In addition, infectious complications, such as breast abscess formations, have also been reported [33].

Although various approaches have been pursued by different centers to detect retained wires before an MRI/ CMR scan, including screening of previous radiological studies, or reviewing the patient's history, in our cohort, multiple chest $\mathrm{x}$-ray images in the anterior posterior view were obtained during the patients' treatment at the intensive care unit and during routine follow up. However, in $53 \%$, retained wires could be detected only on CT or fluoroscopy images.

When analyzing image quality, the impact of retained pacing wires on CMR image quality was negligible. No relevant artifacts could be observed, as the wires neither concealed any pathology nor hampered the use of mapping techniques or post-contrast images. In contrast to residual transvenous PPM and ICD leads, retained temporary pacing wires did not result in any relevant impairment of image quality $[34,35]$. Interrater agreement was good, an excellent agreement was probably not reached as the artifacts caused by the pacing wires could often hardly be detected.

Limitations:

There are limitations of our study that hinder the transition of these results to general radiology. As all CMR examinations were performed on a $1.5 \mathrm{~T}$ according to a standardized protocol, results cannot be easily extrapolated to other protocols that include sequences with a 
higher risk of wire-heating and voltage induction (e.g., diffusion-weighted imaging, prolonged turbo spin echo sequences), other scan areas with different coil settings, or other field strengths. Other limitations were the relatively small sample size and the fact that ECG rhythms were observed for arrhythmias during CMR, but could not be systematically analyzed subsequently. However, most of the patients in this analysis underwent multiple uneventful CMR.

\section{Conclusions}

Repetitive CMR at $1.5 \mathrm{~T}$ in patients with retained temporary right atrial and right ventricular pacing wires after heart transplantation or cardiac surgery appears to be safe and delivers preserved image quality. Therefore, CMR at $1.5 \mathrm{~T}$ should be offered to these patients without restrictions.

\begin{abstract}
Abbreviations
2Ch: Two chamber; 3Ch: Three chamber; 4Ch: Four chamber; CMR: Cardiovascular magnetic resonance; CT: Computed tomography; ECG: Electrocardiogram; HASTE: Half Fourier single-shot turbo spin-echo; ICD: Implanted cardiodefibrillator; LGE: Late gadolinium enhancement; MRI: Magnetic resonance imaging; PM: Pacemaker; PPM: Permanent pacemaker; RA: Right atrium/right atrial; RF: Radiofrequency; RV: Right ventricle/right ventricular; SAR: Specific absorption rate; SAx: Short axis; STIR: Short tau inversion recovery.
\end{abstract}

\section{Acknowledgements}

Not applicable.

\section{Authors' contributions}

DB conceptualized and designed the study. DB and CG analyzed and interpreted the patient data and drafted the manuscript. MS, AK, AZ, MK, CL were major contributors in writing the manuscript. All authors read and approved the final manuscript.

\section{Funding}

This research received no external funding.

\section{Availability of data and materials}

The datasets used and/or analysed during the current study are available from the corresponding author on reasonable request.

\section{Ethics approval and consent to participate}

The study was approved by the institutional review board of the Medical University of Vienna (IRB Nr.: 2012/2015). All the subjects included gave written informed consent prior to study inclusion.

\section{Consent for publication}

Not applicable.

\section{Competing interests}

The authors declare that they have no competing interests.

\footnotetext{
Author details

${ }^{1}$ Department of Biomedical Imaging and Image-guided Therapy, Division of Cardiovascular and Interventional Radiology, Medical University of Vienna, Währinger Gürtel 18-20, 1090 Vienna, Austria. ${ }^{2}$ Department of Medicine II, Division of Cardiology, Medical University of Vienna, Vienna, Austria. ${ }^{3}$ Department of Surgery, Division of Cardiac Surgery, Medical University of Vienna, Vienna, Austria. ${ }^{4}$ Department of Medicine III, Division of Endocrinology and Metabolism, Medical University of Vienna, Vienna, Austria. ${ }^{5}$ Department
}

of Biomedical Imaging and Image-Guided Therapy, Medical University of Vienna, High-Field MR Centre, Vienna, Austria.

Received: 10 November 2020 Accepted: 3 February 2021

Published online: 15 March 2021

\section{References}

1. Reade MC. Temporary epicardial pacing after cardiac surgery: a practical review: Part 2: Selection of epicardial pacing modes and troubleshooting. Anaesthesia. 2007;62:364-73.

2. Elmistekawy E, Gee YY, Une D, Lemay M, Stolarik A, Rubens FD. Clinical and mechanical factors associated with the removal of temporary epicardial pacemaker wires after cardiac surgery. J Cardiothorac Surg. 2016;11:4-8.

3. Ferris NJ, Kavnoudias H, Thiel C, Stuckey S. The 2005 Australian MRI safety survey. Am J Roentgenol. 2007;188:1388-94.

4. Samar H, Yamrozik JA, Williams RB, Doyle M, Shah M, Bonnet CA, et al. Diagnostic value of MRI in patients with implanted pacemakers and implantable cardioverter-defibrillators across a cross population: does the benefit justify the risk? A proof of concept study. JACC Clin Electrophysiol. 2017;3:991-1002

5. Leiner T, Bogaert J, Friedrich MG, Mohiaddin R, Muthurangu V, Myerson S, et al. (2020) on clinical indications for cardiovascular magnetic resonance. J Cardiovasc Magn Reson. 2020;22:76.

6. Erlebacher JA, Cahill PT, Pannizzo F, Knowles RJR. Effect of magnetic resonance imaging on DDD pacemakers. Am J Cardiol. 1986:12:8.

7. Kanal E. Safety of MR imaging in patients with retained epicardial pacer wires. Am J Roentgenol. 1998;170:213-4.

8. Luechinger R, Zeijlemaker VA, Pedersen EM, Mortensen P, Falk E, Duru $F$, et al. In vivo heating of pacemaker leads during magnetic resonance imaging. Eur Heart J. 2005;26:376-83.

9. Langman DA, Goldberg IB, Finn JP, Ennis DB. Pacemaker lead tip heating in abandoned and pacemaker-attached leads at 1.5 tesla MRI. J Magn Reson Imaging. 2011;33:426-31.

10. Pfeil A, Drobnik S, Rzanny R, Aboud A, Böttcher J, Schmidt P, et al. Compatibility of temporary pacemaker myocardial pacing leads with magnetic resonance imaging: an ex vivo tissue study. Int J Cardiovasc Imaging Springer. 2012;28:317-26.

11. Blissett S, Chetrit M, Kovacina B, Mardigyan V, Afilalo J. Performing cardiac magnetic resonance imaging in patients with cardiac implantable electronic devices: a contemporary review. Can J Cardiol Canadian Cardiovascular Society. 2018;34:1682-6.

12. Luetkens JA, Isaak A, Naehle CP, Dabir D, Thomas D. Extended cardiac magnetic resonance imaging with retained temporary transmyocardial pacing lead. Int J Cardiovasc Imaging Springer Netherlands. 2019;35:663-4.

13. Hartnell GG, Spence L, Hughes LA, Cohen MC, Saouaf R, Buff B. Safety of MR imaging in patients who have retained metallic materials after cardiac surgery. Am J Roentgenol. 1997;168:1157-9.

14. Austin CO, Landolfo K, Parikh PP, Patel PC, Venkatachalam KL, Kusumoto FM. Retained cardiac implantable electronic device fragments are not associated with magnetic resonance imaging safety issues, morbidity, or mortality after orthotopic heart transplant. Am Heart J. 2017;190:46-53.

15. Kalb B, Indik JH, Ott P, Martin DR. MRI of patients with implanted cardiac devices. J Magn Reson Imaging. 2018:47:595-603.

16. Rajiah P, Kay F, Bolen M, Patel AR, Landeras L. Cardiac magnetic resonance in patients with cardiac implantable electronic devices: challenges and solutions. J Thorac Imaging. 2020;35:W1-17.

17. Kramer CM, Barkhausen J, Bucciarelli-Ducci C, Flamm SD, Kim RJ, Nagel E. Standardized cardiovascular magnetic resonance imaging (CMR) protocols: 2020 update. J Cardiovasc Magn Reson. 2020;22:1-18.

18. Kalin R, Stanton MS. Current clinical issues for MRI scanning of pacemaker and defibrillator patients. PACE. 2005;5:326-8.

19. Von Knobelsdorff-Brenkenhoff F, Schulz-Menger J. Role of cardiovascular magnetic resonance in the guidelines of the European Society of Cardiology. J Cardiovasc Magn Reson. 2016;18:1-18.

20. Kim J, Hwang J, Choi JH, Choi HI, Kim MS, Jung SH, et al. Frequency and clinical impact of retained implantable cardioverter defibrillator lead materials in heart transplant recipients. PLoS ONE. 2017;12:1-9. 
21. Vermes E, Pantaléon C, Auvet A, Cazeneuve N, Machet MC, Delhommais $A$, et al. Cardiovascular magnetic resonance in heart transplant patients: diagnostic value of quantitative tissue markers: T2 mapping and extracellular volume fraction, for acute rejection diagnosis. J Cardiovasc Magn Reson. 2018;20:1-11.

22. Homsi R, Mellert F, Luechinger R, Thomas D, Doerner J, Luetkens J, et al. Safety and feasibility of magnetic resonance imaging of the brain at 15 tesla in patients with temporary transmyocardial pacing leads. Thorac Cardiovasc Surg. 2019:67:86-91.

23. Kanal E. Standardized approaches to mr safety assessment of patients with implanted devices. Magn Reson Imaging Clin N Am. 2020;7:89.

24. Kappus S, Haber T, Ismer B, Laubenberger J. Qualitative in-vitro-investigation of the MRI compatibility of temporary myocardial electrodes and pacemaker leads. Forsch im Fokus. 2015;12:130-3.

25. Park SM, Kamondetdacha R, Nyenhuis JA. Calculation of MRI-induced heating of an implanted medical lead wire with an electric field transfer function. J Magn Reson Imaging. 2007;26:1278-85.

26. Bonnemains L, Barbier T, Felblinger J. Metal wires should not be abandoned inside implantable cardioverter-defibrillators leads during heart transplantation! Transpl Int. 2016;29:1136-8.

27. Wirtz S, Schulte HD, Winter J, Godehardt E, Kunert J. Reliability of different temporary myocardial pacing leads. Thorac Cardiovasc Surg. 1989;37:163-8

28. Peden CJ, Collins AG, Butson PC, Whitwam JG, Young IR. Induction of microcurrents in critically ill patients in magnetic resonance systems. Crit Care Med US. 1993;21:1923-8.

29. Levine GN, Gomes AS, Arai AE, Bluemke DA, Flamm SD, Kanal E, et al. Safety of magnetic resonance imaging in patients with cardiovascular devices: An American heart association scientific statement from the committee on diagnostic and interventional cardiac catheterization, council on clinical cardiology, and the council o. Circulation. 2007;116:2878-91.

30. Murphy KJ, Cohan RH, Ellis JH. MR imaging in patients with epicardial pacemaker wires. Am J Roentgenol. 1999;172:727-8.

31. Shaikhrezai K, Khorsandi M, Patronis M, Prasad S. Is it safe to cut pacing wires flush with the skin instead of removing them? Interact Cardiovasc Thorac Surg. 2012;15:1047-51.

32. Horng GS, Ashley E, Balsam L, Reitz B, Zamanian RT. Progressive dyspnea after CABG: complication of retained epicardial pacing wires. Ann Thorac Surg. 2008;86:1352-4

33. Jafferjee N, Li B, Mijangos S, Thu Ma AM, Manghisi S. Migrated abandoned epicardial pacing wire resulting in a breast abscess. Breast J. 2014;20:200-1.

34. Klein-Wiele O, Garmer M, Busch M, Mateiescu S, Urbien R, Barbone G, et al. Cardiovascular magnetic resonance in patients with magnetic resonance conditional pacemaker systems at $1.5 \mathrm{~T}$ : influence of pacemaker related artifacts on image quality including first pass perfusion, aortic and mitral valve assessment, flow measurement. Int J Cardiovasc Imaging 2017;33:383-94.

35. Hilbert S, Jahnke C, Loebe S, Oebel S, Weber A, Spampinato R, et al. Cardiovascular magnetic resonance imaging in patients with cardiac implantable electronic devices: A device-dependent imaging strategy for improved image quality. Eur Heart J Cardiovasc Imaging. 2018;19:1051-61.

\section{Publisher's note}

Springer Nature remains neutral with regard to jurisdictional claims in published maps and institutional affiliations.
Ready to submit your research? Choose BMC and benefit from:

- fast, convenient online submission

- thorough peer review by experienced researchers in your field

- rapid publication on acceptance

- support for research data, including large and complex data types

- gold Open Access which fosters wider collaboration and increased citations

- maximum visibility for your research: over $100 \mathrm{M}$ website views per year

At BMC, research is always in progress.

Learn more biomedcentral.com/submissions 